\title{
Advective flow in spherical floc
}

\author{
Z. Yang ${ }^{a}$, X.F. Peng ${ }^{a}$, D.J. Lee ${ }^{b, *}$, S. Ay ${ }^{c}$ \\ a Department of Thermal Engineering, Tsinghua University, Beijing 100084, People's Republic of China \\ b Department of Chemical Engineering, National Taiwan University, Taipei 10617, Taiwan \\ ${ }^{c}$ Department of Mechanical Engineering \& Fuel Cells Research Center, Yuan Ze University, Chungli 320, Taiwan
}

Received 5 September 2006; accepted 11 January 2007

Available online 18 January 2007

\begin{abstract}
Numerous structural models of flocs, such as homogeneous model or radially-varying model, were proposed in literature for predicting the extent of advective flow on the intrafloc transport processes. This work probed the three-dimensional structure of original and chemically flocculated wastewater flocs using the fluorescence in situ hybridization (FISH) and the confocal laser scanning microscope (CLSM) techniques, from which the spherical mesh model on real floc structure was constructed. Simulation results revealed that if an average characteristic of sludge floc, such as porosity or drag force correction factor of sludge floc is of concern, both homogeneous or radially-varying models may be able to apply, particularly for those flocs that were closely compacted. However, the detailed flow patterns inside real floc are much more tortuous than those of the homogeneous or radially-varying models. If local hydrodynamic environment within the floc is of interest, then only the complicated structural model with real floc could be applicable.
\end{abstract}

(c) 2007 Elsevier Inc. All rights reserved.

Keywords: Floc model; Advective flow; FISH; CLSM

\section{Introduction}

Sludge flocs are regarded as highly porous fractal-like aggregates composed by many particles [1]. Numerous structural models of flocs have been proposed to estimate the effects of advective flow on the intrafloc transport processes. Model considering the floc's interior as a uniform porous medium is referred to as the "homogeneous model" [2-13]. The model taking the floc as a porous medium of radiallyvarying porosity (and permeability as well) [14-21] is named as "radially-varying model." In some relevant works, the floc was regarded as of a self-similar, fractal-like structure [22-27]. Solving the equations of motion with pre-described floc structure could derive the advective flow field. However, up to now no comparisons between the outputs of various floc models were made since there exists no common basis to compare with.

\footnotetext{
* Corresponding author. Fax: +88622362 3040

E-mail address: djlee@ntu.edu.tw (D.J. Lee).
}

Our group detailed the floc's interior structure by employed the fluorescence in situ hybridization (FISH) technique and confocal laser scanning microscope (CLSM), and subsequently, established the three-dimensional floc model for biological flocs [28-32]. A highly tortuous, inter-connected pore networks were noticeable for the floc interior. It is not clear whether the existing floc models can properly describe the complicated, intra-flow flow fields for real biological flocs.

This study conducted experimental works on probing interior structure of wastewater sludge floc using CLSM imaging. Then the outputs from homogeneous models and radiallyvarying models were compared with the results by real floc. The three-dimensional interior structure of wastewater sludge floc was probed and reconstructed into a spherical shape (the constructed model). The homogeneous and radially-varying models with the same floc porosity and diameter of the constructed model floc were also established. Then the advective flow field through the two models and the real floc model was simulated and compared. This work made no comparisons with the intrafloc flow fields using fractal-like models. 


\section{Experimental}

\subsection{The samples}

Waste activated sludge was taken from the Min-Sheng $\mathrm{Mu}-$ nicipal Wastewater Treatment Plant in Taipei, which was gravitationally settled to a solid content of around $15,000 \mathrm{mg} \mathrm{L}^{-1}$, and was the testing sample for the present test. The chemical oxygen demand (COD) for the sludge was $24,400 \mathrm{mg} \mathrm{L}^{-1}$, obtained from direct reading spectrometer (DR/2000, HACH, USA). Dry solids density of sludge was measured by an Accupyc Pycnometer 1330 (Micromeritics) as $1450 \mathrm{~kg} \mathrm{~m}^{-3}$. The original floc (\#A) was sampled from the original sludge.

Some sludge samples were flocculated by the cationic flocculant, a polyacrylamide indicated as polymer T-3052 from Kai-Guan Inc., Taiwan. The polymer T-3052 has an average molecular weight of 107 daltons (Da) and a charge density of $20 \%$. The polymer solution was gradually poured into the mixing vessel containing fresh sludge samples with $200 \mathrm{rpm}$ of stirring for $5 \mathrm{~min}$ (rapid mix) followed by $50 \mathrm{rpm}$ of another 20 min (slow mix) [33]. Based on filterability tests (data not shown), the "optimal" dose of polyelectrolyte for the present sludge was noted $40 \mathrm{ppm}$, and was used as the dose adopted herein. The "flocculated flocs" \#B and \#C were sampled from the flocculated sludge thus produced.

\subsection{FISH and CLSM}

The present work employed the CLSM (OLYMPUS BX50, Japan) equipped with an image processor (OLYMPUS FV5 PSU, Japan) and an argon laser source to stimulate the fluorescence. The sludge floc was imaged with a $10 \times$ objective with the software FLUOVIEW version 3.0. The microscope scanned the samples at fixed depth and digitized the image obtained. Sludge flocs for CLSM analysis were first fixed with $3 \%$ paraformadehyde in phosphate-buffered saline (PBS). Then the fixed sample was embedded in low-melting-point agar (with melting point of $75^{\circ} \mathrm{C}$ and gelling point of $38^{\circ} \mathrm{C}$ ) for the FISH. In this study, we used the probe, EUB338 (labeled by rhodamine) and ARCH915 (labeled by tetrachlorofluorescein), for detection of members of domain Bacteria with high cellular ribosome content and those that cannot be detected by EUB338.
The stained samples were washed three times to remove extra probes by hybridization buffer solution.

During the acquisition of CLSM images, selecting appropriate vision field in order to collect the structural information for entire floc accommodated accommodation of entire floc. More than 350 CLSM images were sampled for a floc.

\subsection{Image processing}

Irregular outer layer of floc model was removed to make a perfect spherical shape as assumed by the homogeneous and radially-varying models (Fig. 1). Then the image-thresholding algorithm, Otsu's method [34-36], determined the threshold values of each sliced images (Fig. 1a). After the boundaries between solids and void space were allocated, the marching cubes algorithm was used to patch the sphere's surface with triangle iso-surfaces. Finally, the advancing-front algorithm was applied to fill each region defined by the simplified polygonal surface model of pores, with unstructured tetrahedral volumetric grids. The tetrahedral quantities of the generated volumetric grids were further simplified to comply with the data format for fluid dynamic software. Software Amira 3.0 was used to construct the thresholded sliced images as iso-surfaces (polygonal surface models and volumetric grids) (Fig. 1c).

Three floc models were examined, including one original floc (\#A, diameter of $106 \mu \mathrm{m}$ and porosity of 0.61 ) and two chemically flocculated flocs (\#B, diameter of $195 \mu \mathrm{m}$ and porosity of 0.54 ; and $\# \mathrm{C}$, diameter of $132 \mu \mathrm{m}$ and porosity of 0.51$)$.

\section{Models and solution}

We simulated the flow fields surrounding and inside a model floc subjected to an incoming unbound Newtonian fluid. The fluid of density $\rho$ and viscosity $\mu$ is flowing at a uniform velocity of $\vec{u}_{\infty}$ from infinity toward the fixed floc. Fig. 2 depicts schematically the flow process. The diameter $\left(D_{\mathrm{L}}\right)$ and the length $(L)$ of the domain tube were kept 160 times of the floc diameter $\left(d_{\mathrm{f}}=2 R_{\mathrm{f}}\right)$ to minimize boundary effects. Preliminary calculations revealed that the fluid field can approximate an unbound flow field if both $D_{\mathrm{L}} / d_{\mathrm{f}}$ and $L / d_{\mathrm{f}}>60$.

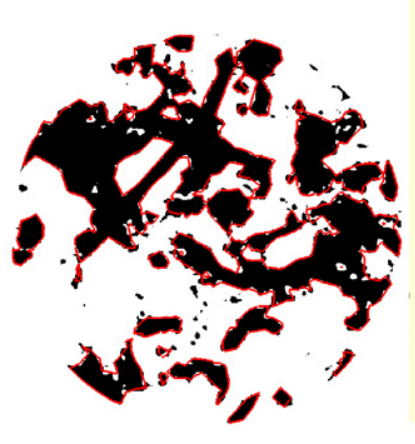

(a)

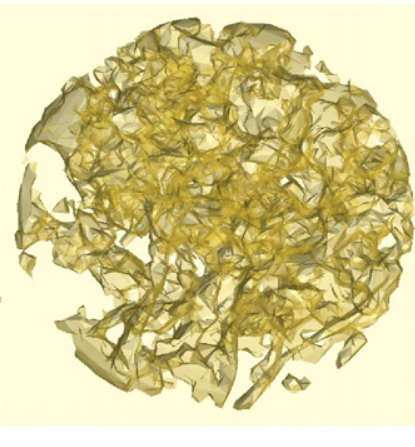

(b)

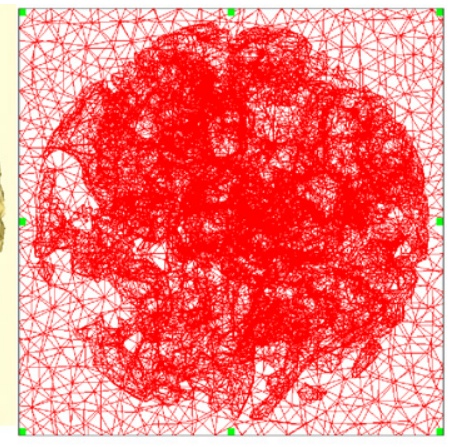

(c)

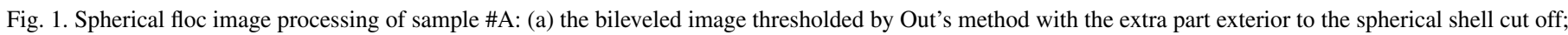
(b) three-dimensional constructed spherical floc; (c) tetrahedral volumetric grids exterior and interior to the floc. 


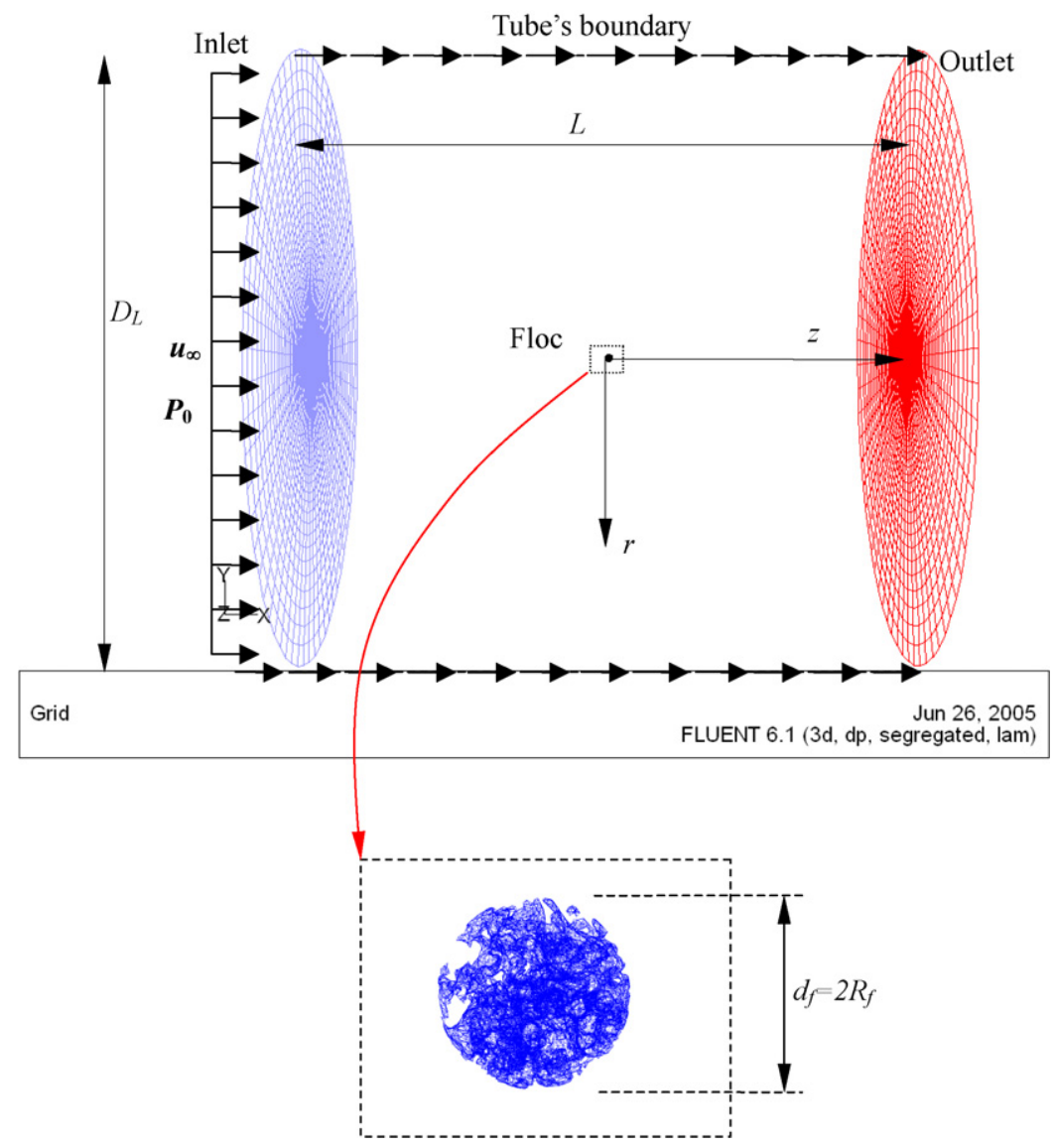

Fig. 2. The computational domain adopted in present study to simulate an infinite flow field approaching a floc. $L / d_{\mathrm{f}}=160, D_{\mathrm{L}} / d_{\mathrm{f}}=160$.

\subsection{Constructed model}

The intrafloc flow field for the present constructed, spherical floc model (Fig. 1c) was obtained by solving Eq. (1) by taking the surrounding fluid and intrafloc fluid as a continuum without artificially creating an interface between the special boundary of entire floc and the surrounding fluid:

$\left(\vec{u}^{\prime} \cdot \nabla\right) \vec{u}^{\prime}+\frac{P_{0}}{\rho V^{2}} \nabla P^{\prime}=\frac{1}{\operatorname{Re}} \nabla^{2} \vec{u}^{\prime}$,

where $\vec{u}^{\prime}=\vec{u} / u_{\infty}$ and $P^{\prime}=P / P_{0}$. The corresponding boundary conditions are as follows:

$\vec{u}=\overrightarrow{1} \quad$ at outer tube's surfaces,

$\frac{\partial \vec{u}}{\partial r}=0 \quad$ at $r=0$ (outside floc),

$\vec{u}=\overrightarrow{0} \quad$ at rugged solid surface inside floc.

\subsection{Radially-varying model}

The radially-varying model adopts non-constant distribution of porosity (and hence permeability) that changes radially in the floc. The overall porosity for the entire floc can be related to the floc diameter $d_{\mathrm{f}}$ and an exponent $(n)$ as follows [16,37,38]:

$\varepsilon=1-\left(\frac{d_{\mathrm{p}}}{d_{\mathrm{f}}}\right)^{n}$

with local porosity function as follows:

$$
\begin{array}{ll}
\varepsilon(r)=1-\left(\frac{d_{\mathrm{p}}}{2 r}\right)^{n}, & d_{\mathrm{p}} / 2<r<d_{\mathrm{f}} / 2, \\
\varepsilon(r)=0, & r<d_{\mathrm{p}} / 2,
\end{array}
$$

where $d_{\mathrm{p}}$ is the diameter of the primary particles. Note that Eq. (3) is not an expression for fractal models, but that for radially-varying model with two parameters: $d_{\mathrm{p}}$ and $n$. An impermeable solid core of diameter $d_{\mathrm{p}}$ exists at the center of the floc. Then by taking the floc porosity and the floc diameter from the constructed floc model as input parameters, Eq. (3) then relates primary particle diameter and the exponent $n$. Restated, only one parameter is fixed for system specification. Since the porosity of the studied floc models was fixed as that measured by real flocs, a high $n$ means a porosity distribution significantly deviating from a uniform distribution.

For the radius-varying floc model, the point permeability of the floc can be estimated based on given theoretical model, such as the following Happel's model:

$K_{i}=\frac{d_{\mathrm{p}}^{2}}{18 \gamma^{3}}\left(3-\frac{9 \gamma}{2}+\frac{9 \gamma^{5}}{2}-3 \gamma^{6}\right) /\left(3+2 \gamma^{5}\right)$,

where $\gamma=1-\left(2 r / d_{\mathrm{p}}\right)^{-n / 3}$. Equation is valid in a shell of radially-varying model in which the porosity is a constant (Eq. (4)). The volume-average permeability of the floc is evaluated as follows:

$K_{\mathrm{h}}=\frac{1}{V} \int_{V} K_{i} \mathrm{~d} V$, 
where $V$ is the volume of the floc sphere.

In simulation, the floc interior is equally divided into 20 layers of thickness of $\left(R_{\mathrm{f}} / 20\right)$, with the intra-layer fluid fields calculated by the Darcy-Brinkman equation:

$\vec{u}_{i}^{\prime}+\frac{\mathrm{Eu} \cdot \mathrm{Re}}{\beta_{i}^{2}} \nabla P^{\prime}=\frac{1}{\beta_{i}^{2}} \nabla^{2} \vec{u}_{i}^{\prime}$,

where $\vec{u}_{i}^{\prime}=\vec{u}_{i} / u_{\infty}, \mathrm{Eu}=P_{0} / \rho u_{\infty}^{2}, \operatorname{Re}=u_{\infty} d_{\mathrm{f}} / \nu$, and $\beta_{i}=$ $\left(d_{\mathrm{f}} / 2\right) / K_{i}^{0.5}$. The boundary conditions at the interface between two adjacent layers $(i)$ and $(i+1)$ are as follows:

$\vec{u}_{i}=\vec{u}_{i+1}, \quad i=1-19$,

$\nabla \vec{u}_{i}=\nabla \vec{u}_{i+1}, \quad i=1-19$.

The boundary conditions at floc's outer surface are:

$\vec{u}=\vec{u}_{20}$,

$\nabla \vec{u}=\nabla \vec{u}_{20}$.

While at the floc's center,

$\frac{\partial \vec{u}_{1}}{\partial r}=0$.

The floc diameter and floc porosity were fixed for each model floc with exponent $n$ being used as an adjustable parameter. Once the $n$ value was prescribed, the diameter of primary particle (Eq. (3)) and then permeability (Eq. (5)) for radially-varying model could be calculated. Governing equations, Eqs. (1) and (7), were then solved with boundary conditions (Eqs. (2a) and (2b) and (8a)-(8e)) to get the interfloc and intrafloc fluid fields.

\subsection{Homogeneous models}

In simulation with homogeneous model, the permeability is taken as a constant determined by Eq. (6) for comparison sake.
The inter- and intrafloc flow fields were calculated using Eq. (1) and the following Darcy-Brinkman equation:

$\vec{u}_{\mathrm{f}}^{\prime}+\frac{\mathrm{Eu} \cdot \operatorname{Re}}{\beta^{2}} \nabla P^{\prime}=\frac{1}{\beta^{2}} \nabla^{2} \vec{u}_{\mathrm{f}}^{\prime}$

where $\vec{u}_{\mathrm{f}}^{\prime}=\vec{u}_{\mathrm{f}} / u_{\infty}$ and $\beta=\left(d_{\mathrm{f}} / 2\right) / K_{\mathrm{h}}^{0.5}$. The boundary conditions used were as follows:

$\vec{u}=\vec{u}_{\mathrm{f}} \quad$ on floc's surface,

$\nabla \vec{u}=\nabla \vec{u}_{\mathrm{f}} \quad$ on floc's surface,

$\frac{\partial \vec{u}_{\mathrm{f}}}{\partial r}=0 \quad$ at floc center.

\subsection{Numerical solution}

The tetrahedral grids in computational domain were first preprocessed using geometry modeling mesh generation software, GAMBIT 2.0 (Fluent Inc., USA). Then the governing equations were solved using software, FLUENT 6.1 (Fluent Inc., USA). The pressure-velocity coupling algorithm was SIMPLEC (Semi-Implicit Method for Pressure-Linked EquationsConsistent). The calculation was performed at a maximum relative error of $0.01 \%$. In the present work, $\operatorname{Re}=0.1$.

\section{Results and discussion}

\subsection{Floc porosity}

For homogeneous model, porosity is a constant regardless of the radial position in floc. The porosity over the five equally divided shells of constructed and radially-varying models for the floc \#A were compared in Fig. 3, with local porosities averaged over five equally-divided shells in the floc. The homogeneous model yields a horizontal line of average porosity in Fig. 3.

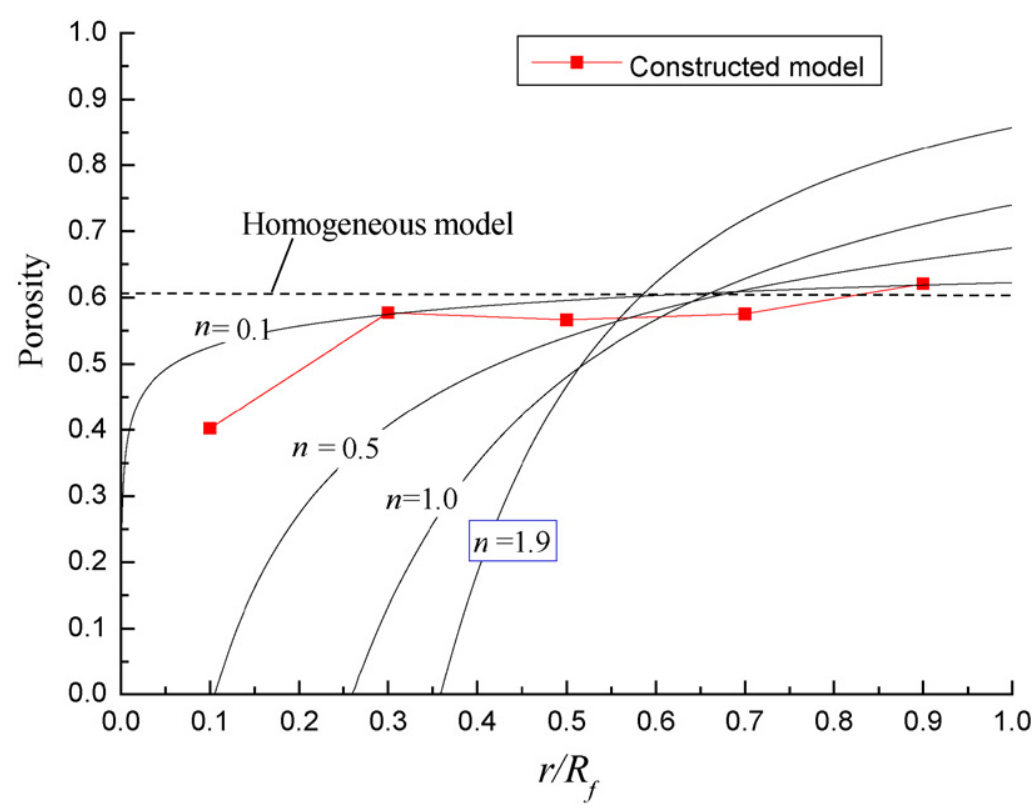

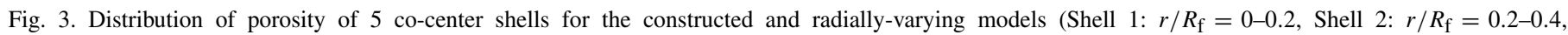
Shell 3: $r / R_{\mathrm{f}}=0.4-0.6$, Shell 4: $r / R_{\mathrm{f}}=0.6-0.8$, Shell 5: $\left.r / R_{\mathrm{f}}=0.8-1.0\right)$. Floc \#A $\left(\varepsilon=0.61\right.$ and $\left.d_{\mathrm{f}}=106 \mu \mathrm{m}\right)$. 
The porosity distribution (averaged over the local shells) of the constructed model is noted rather smooth over floc radius, characterizing by models with $n=0.2-0.3$. Restated, in the sense of porosity distribution, the interior structure would be preferably represented by a homogeneous rather than a radially-varying model.

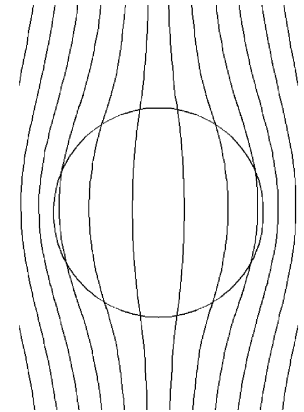
$n=1.9(\mathrm{H})$

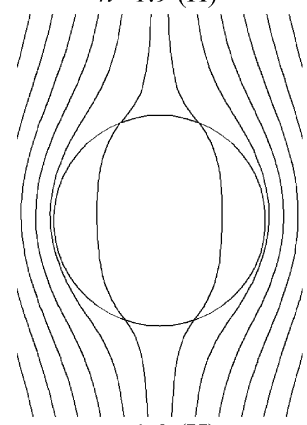
$n=1.0(\mathrm{H})$

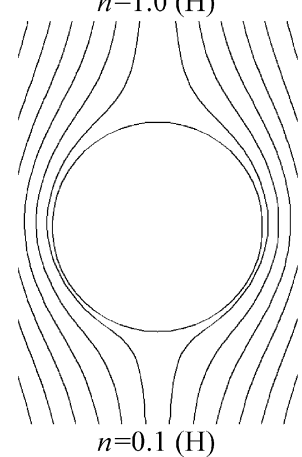

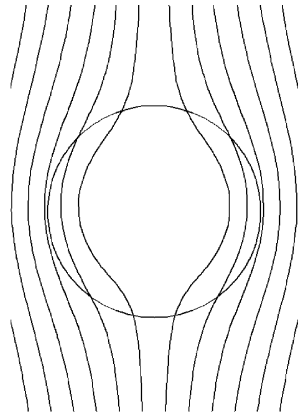
$n=1.9(\mathrm{~F})$

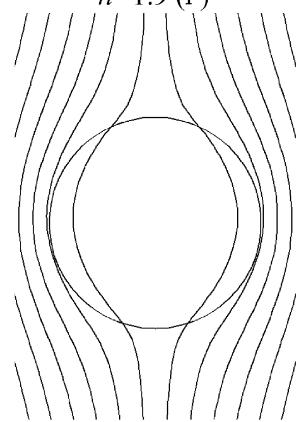
$n=1.0(\mathrm{~F})$

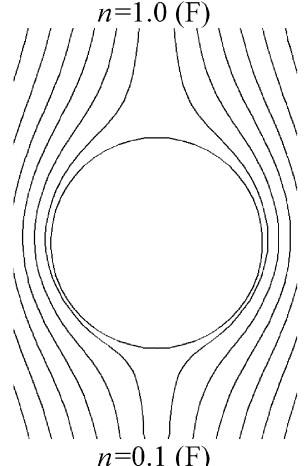

Fig. 4. Stream lines through a porous sphere with the same overall porosity (0.61) and diameter $(106 \mu \mathrm{m})$ as for floc \#A. Re $=0.1$. $(\mathrm{H})$ Homogeneous model; $(\mathrm{F})$ radially-varying model.
In the following demonstration studies, the volume-average porosities of all tested models were set identical for the same model floc for comparison sake.

\subsection{Advective flow}

Fluid tends to flow through the outer layers of radiallyvarying model floc owing to the relatively higher permeability compared with the inner core. At low- $n$ case $(n=0.1$ in Fig. 4), the flow fields approach those of an impermeable sphere (Stokes' law). However, the difference between the homogeneous and radially-varying models grows at high- $n$ case $(n=1.9$ in Fig. 4$)$.

The advective flow pattern was displayed by the intrafloc streamlines of the spherical, constructed model floc (Fig. 1c), with incoming fluid flowing along the $x$-, $y$-, and $z$-directions (Figs. 5a-5c), colored by the static pressure. The streamlines of constructed model (floc \#A) are in all cases much more tortuous than those of the homogeneous or radially-varying models (Fig. 4), yielded by the complicated, inter-connected floc pores with rugged surfaces.

The radial distributions of the mean axial velocity for the three studied models, normalized by their corresponding maximum velocities, were shown in Fig. 6. At low $n$, most fluid flows over the rim regimes of the flocs while the radial distributions of both models are close. At high $n(n=1.9)$, a significant advective flow can be noted for the homogeneous model while the axial velocity vanishes for radially-varying model at $r / R_{\mathrm{f}}<0.5$. Interestingly, regardless of the differences in individual flocs (\#A-\#C, data for \#B and \#C not shown) or of the flow directions ( $x$-, $y$-, or $z$-direction) along the floc, all axial velocity distributions follow closely with the radially-varying model with $n=0.8$ for floc \#A with identical average permeability.

The drag force exerting on the reconstructed model can be calculated by applied the flowing integration over the entire floc surface (including the surface of channel interior to floc),

$\vec{F}=\int_{\text {surf }} \mathrm{d} \vec{A} \cdot \overrightarrow{\vec{\tau}}$,

where $\vec{A}$ is the directional surface area, $\vec{\tau}$ is the force tensor, and "surf" is the surface of floc. While for the porous model,
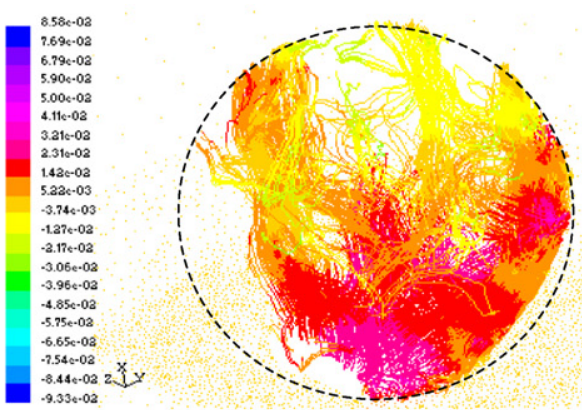

(a)

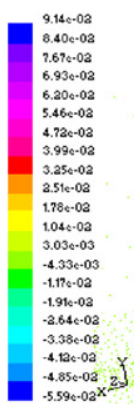

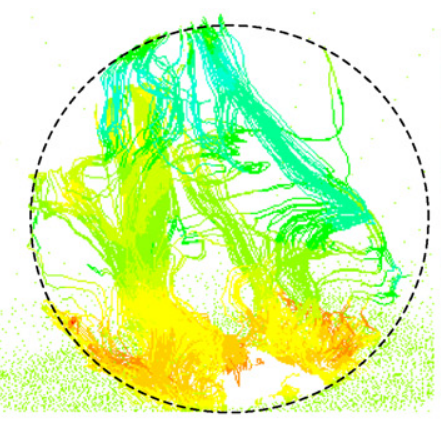

(b)

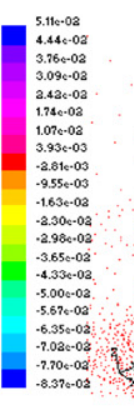

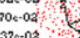

Fig. 5. The streamlines of intrafloc flow in floc \#A subjected to the incoming flow in the (a) $x$-direction; (b) $y$-direction; and (c) $z$-direction, colored by static pressure (Pa, gauge). 

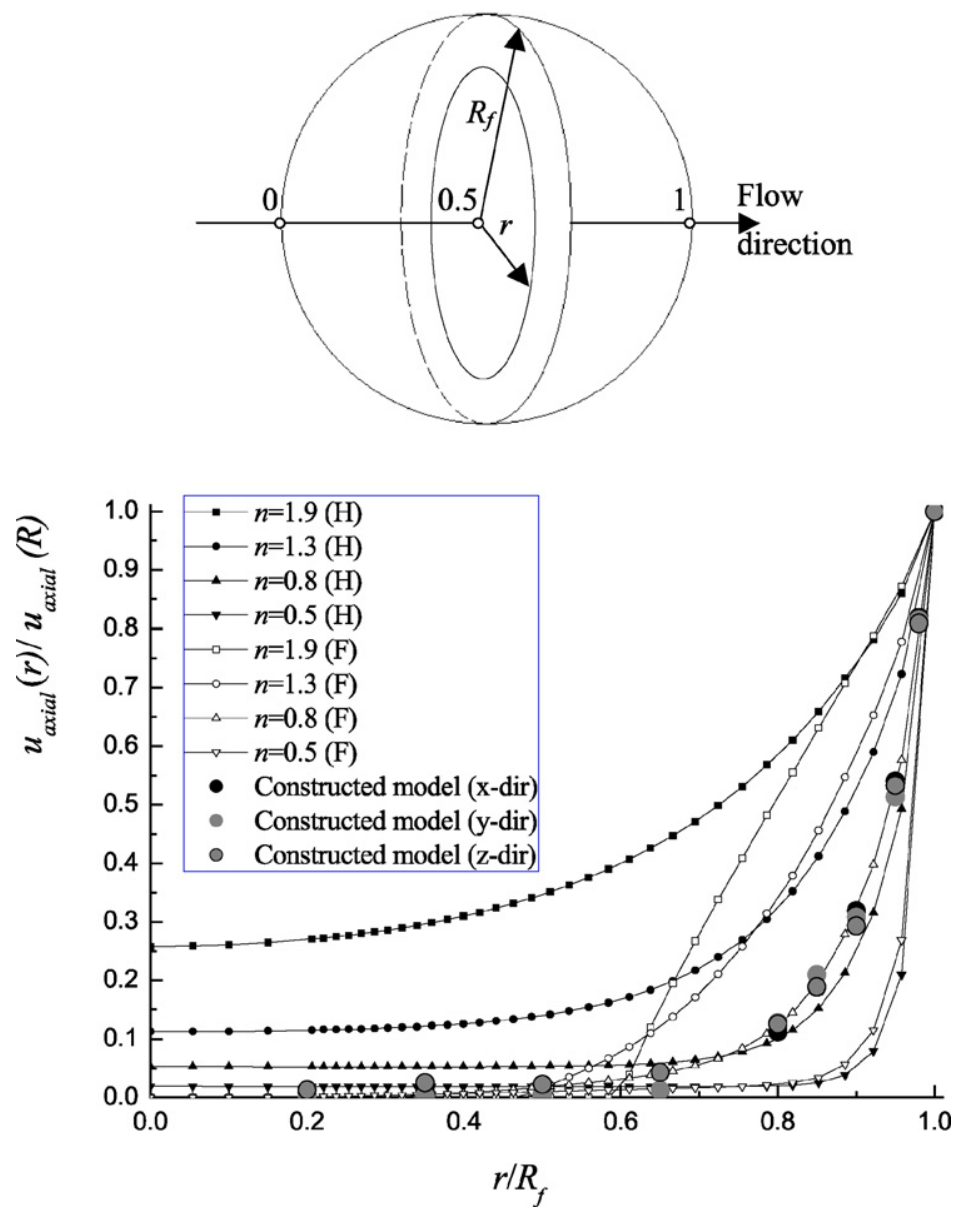

Fig. 6. Radially dependent low-directional velocity, $u_{\mathrm{axial}}$, in the middle plane of floc perpendicular to the flow-direction, normalized by the velocity at $R_{\mathrm{f}}, u_{\mathrm{R}}$, for the constructed, homogeneous $(\mathrm{H})$ and radially-varying $(\mathrm{F})$ models. Floc \#A $\left(\varepsilon=0.61\right.$ and $\left.d_{\mathrm{f}}=106 \mu \mathrm{m}\right)$.

this integration is done on the spherical surface of the porous region. With the force acquired, the drag factor, $\Omega$, can be calculated as

$$
\Omega=\frac{|F|}{6 \pi R_{\mathrm{f}} \mu u_{\infty}} .
$$

Fig. 7 shows the $\Omega$ values for the three models while the exponent in radially-varying model is used as an adjustable parameter. The $\Omega$ values for the constructed models floc \#A-\#C were calculated as 0.961, 0.981, and 0.983, respectively. As $n$ is increased, the corresponding $\Omega$ value decreases accordingly. The radially-varying model predicts a lower drag force than does the homogeneous model. Over appropriate parametric range, the radially-varying and the homogeneous model produce similar $\Omega$ values from constructed model. The closeto-unity $\Omega$ value indicates that the flocs investigated herein experience a drag force similar to an impermeable sphere of the same diameter.

\subsection{Discussions}

As mentioned above, the porosity distribution (average over shells) in constructed floc is rather smooth over floc radius, and can hence be approximated by a radially-varying model of $n=$ $0.2-0.3$, or a homogeneous model of identical average poros- ity (Fig. 3). However, the streamlines of constructed model are much more tortuous (Fig. 5) than those of the homogeneous or radially-varying models (Fig. 4). Meanwhile, the radiallyvarying model or homogeneous model of $n=0.3-0.4$ predicts the drag force experienced by the model flocs. However, considering the axial velocity distributions averaged over shells over the floc's cross-section were best described by radially-varying model with $n=0.7-0.8$.

Neither the radially-varying nor homogeneous models predict the $\theta$-(angular) dependence intrafloc flow field in flocs. Fig. 8 shows the local velocities for floc \#A at $r / R_{\mathrm{f}}=0.65$ as a demonstration example. Along the angular axis $(\theta=$ $\left.0^{\circ}-360^{\circ}\right)$ the axial velocity $u_{\text {axial }}$ at fixed floc radial position can change significantly (for instance, $\theta=75^{\circ}-95^{\circ}$ in Fig. 8), or even reverses in flow direction (for instance, $\theta=310^{\circ}-360^{\circ}$ in Fig. 8). Certain big pores in the floc interior dominate the intrafloc flow fields. Certain big pores should control most of intrafloc flow.

The following distribution factor, $\alpha$, is defined to quantitatively describe the unevenness of the axial flow distributions at various floc radial positions:

$$
\alpha(r)=\frac{1}{\bar{u}_{\text {axial }}(r)} \sqrt{\frac{\int_{2 \pi}\left(u_{\text {axial }}(r, \theta)-\bar{u}_{\text {axial }}(r)\right)^{2} \mathrm{~d} \theta}{2 \pi}},
$$




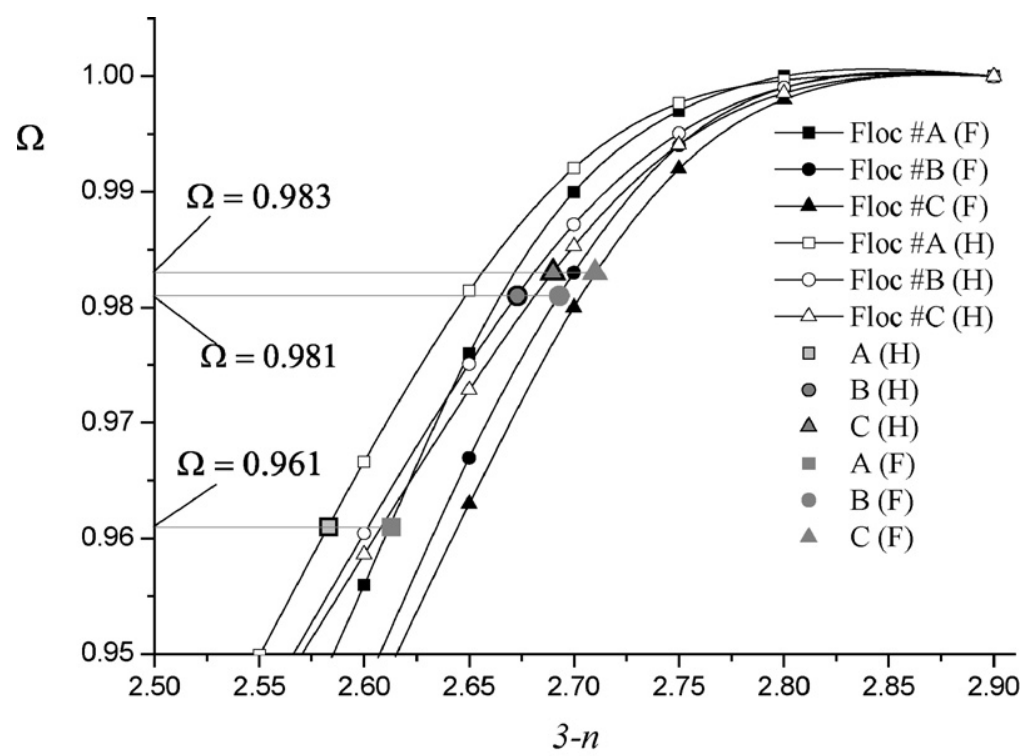

Fig. 7. Calculated $\Omega$ values for radially-varying and homogeneous models with the three model flocs.
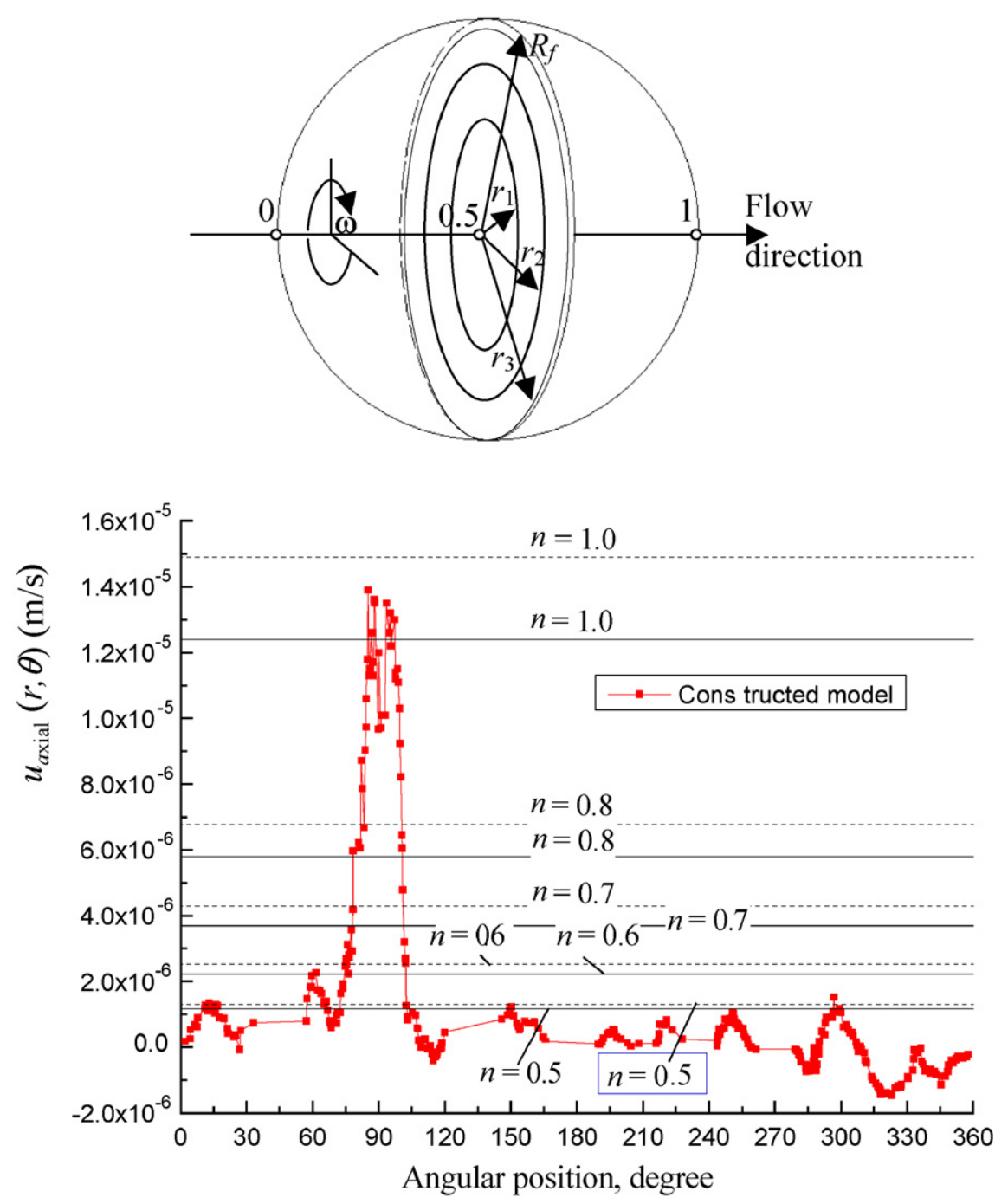

Fig. 8. Angular distribution of flow directional velocity at specific radii of the middle plane of floc \#A. $r / R_{\mathrm{f}}=0.65$. Solid, horizontal lines: radially-varying model; dashed, horizontal lines: homogeneous model. 
Table 1

Values of distribution factor, $\alpha$

\begin{tabular}{|c|c|c|c|c|c|c|c|}
\hline \multirow[t]{2}{*}{ Floc } & \multirow[t]{2}{*}{ Direction } & \multicolumn{6}{|c|}{ Radial position } \\
\hline & & $r / R_{\mathrm{f}}=0.2$ & $r / R_{\mathrm{f}}=0.35$ & $r / R_{\mathrm{f}}=0.5$ & $r / R_{\mathrm{f}}=0.65$ & $r / R_{\mathrm{f}}=0.8$ & $r / R_{\mathrm{f}}=0.95$ \\
\hline \multirow[t]{3}{*}{ \#A } & $x$ & 1.36 & 1.56 & 1.41 & 3.20 & 1.97 & 1.12 \\
\hline & $y$ & 1.91 & 1.09 & 1.73 & 2.08 & 1.53 & 1.12 \\
\hline & $z$ & 0.92 & 1.01 & 0.83 & 1.32 & 1.52 & 0.85 \\
\hline \multirow[t]{3}{*}{ \#B } & $x$ & 0.94 & 2.39 & 1.85 & 2.62 & 2.48 & 1.26 \\
\hline & $y$ & 1.17 & 1.64 & 1.45 & 1.59 & 2.32 & 1.42 \\
\hline & $z$ & 1.02 & 1.61 & 1.39 & 0.99 & 1.03 & 1.08 \\
\hline \multirow[t]{3}{*}{ \#C } & $x$ & 0.92 & 3.00 & 2.10 & 1.90 & 2.02 & 1.29 \\
\hline & $y$ & 1.24 & 1.45 & 2.64 & 2.10 & 3.08 & 1.07 \\
\hline & $z$ & 0.75 & 1.02 & 1.18 & 1.42 & 1.85 & 1.16 \\
\hline
\end{tabular}

where the average axial velocity over angular axis is defined as follows:

$\bar{u}_{\text {axial }}(r)=\frac{\int_{2 \pi} u_{\text {axial }}(r, \theta) \mathrm{d} \theta}{2 \pi}$.

$\alpha=0$ by definition for homogeneous or radially-varying models since their structures are angularly symmetric. A greaterthan-zero $\alpha$ indicates a nonsymmetric distribution of $u_{\text {axial }}$ along angular axis. The $\alpha$ parameters at floc surface regime $\left(r / R_{\mathrm{f}}=0.95\right.$ in Table 1$)$ are all around unity, probably owing to the strong effects of the uniform, surrounding fluid flow. Significant variations in axial velocities occur at the intermediate shell $\left(0.35<r / R_{\mathrm{f}}<0.8\right.$ in Table 1$)$.

Hence, if an average characteristic of sludge floc, such as porosity or drag force correction factor of sludge floc is of concern, both homogeneous or radially-varying models may be fine to apply, particularly for those flocs that compact closely. Meanwhile, if the local environment of cells within the floc is of interest, then neither the homogeneous nor the radially-varying model can describe the very complicated intrafloc field herein investigated.

\section{Conclusions}

This work compared the intra-floc flow fields yielded by two floc models commonly adopted in literature, the homogeneous model and the radially-varying model, with that obtained based on real floc structure constructed by FISH and CLSM techniques. The spherical mesh models on real floc structure were for the first time realized. Fluid tends to flow through the outer layers of homogeneous or radially-varying model floc. However, the advective flow pattern in the constructed spherical model are much more tortuous than those of the homogeneous or radially-varying models, yielded by the complicated, inter-connected floc pores with rugged surfaces. The radiallyvarying model predicts a lower drag force than does the homogeneous model. But both theoretical models can reproduce the drag force by the constructed model with appropriately selected model parameters. When an average characteristic of floc is of concern, such as the average porosity or the overall drag force, both homogeneous or radially-varying models may be able to apply to model the floc process, particularly for those flocs that were closely compacted. However, if the local hydrodynamic environment within the floc is of interest, such as the rate of transport of nutrients and oxygen in the intra-floc microchannels, then only the complicated structural model with real floc should be applicable.

\section{Acknowledgment}

Z. Yang thanks Department of Chemical Engineering at National Taiwan University for inviting him as a visiting student from March to July, 2005.

\section{References}

[1] D.H. Li, J.J. Ganczarczyk, Environ. Sci. Technol. 23 (1989) 1385.

[2] H.C. Brinkman, Appl. Sci. Res. A 1 (1947) 27.

[3] H.C. Brinkman, Appl. Sci. Res. A 1 (1947) 81.

[4] H.C. Brinkman, Research (London) 2 (1949) 190.

[5] G. Ooms, P.F. Mijnleff, H. Beckers, J. Chem. Phys. 53 (1970) 4123.

[6] J.H. Masliyah, G. Neale, Chem. Eng. Sci. 42 (1987) 245.

[7] A.S. Kim, R. Yuan, J. Membr. Sci. 249 (2005) 89.

[8] T. Tao, X.F. Peng, D.J. Lee, J. Chin. Inst. Chem. Eng. 37 (2006) 299.

[9] R.M. Wu, D.J. Lee, Water Res. 32 (1998) 860.

[10] R.M. Wu, D.J. Lee, Chem. Eng. Sci. 54 (1999) 5717.

[11] R.M. Wu, D.J. Lee, Water Res. 35 (2001) 3226.

[12] R.M. Wu, G.W. Tsou, P.S. Yen, D.J. Lee, X.F. Peng, J. Chem. Eng. Jpn. 35 (2002) 540

[13] G.W. Tsou, R.M. Wu, P.S. Yen, D.J. Lee, X.F. Peng, J. Colloid Interface Sci. 250 (2002) 400.

[14] M. Vanni, Chem. Eng. Sci. 55 (2000) 685.

[15] A.S. Kim, R. Yuan, J. Colloid Interface Sci. 285 (2005) 627.

[16] S. Chellam, M.R. Wiesner, Water Res. 27 (1993) 1943.

[17] S. Veerapaneni, M.R. Wiesner, J. Colloid Interface Sci. 177 (1996) 17,745.

[18] J.P. Hsu, Y.H. Hsieh, J. Colloid Interface Sci. 264 (2003) 517.

[19] J.P. Hsu, Y.H. Hsieh, J. Colloid Interface Sci. 275 (2004) 309.

[20] J.P. Hsu, M.C. Li, A.C. Chang, J. Colloid Interface Sci. 284 (2005) 332.

[21] J.P. Hsu, S.J. Yeh, D.J. Lee, J. Colloid Interface Sci. 292 (2005) 290.

[22] X.Y. Li, B.E. Logan, Water Res. 35 (2001) 3373.

[23] X.Y. Li, B.E. Logan, Environ. Sci. Technol. 31 (1997) 1229.

[24] X.Y. Li, B.E. Logan, Environ. Sci. Technol. 31 (1997) 1237.

[25] X.Y. Li, R.P.C. Leung, Environ. Sci. Technol. 39 (2005) 2731.

[26] X.Y. Li, Y.A. Yuan, Water Res. 36 (2002) 3110.

[27] X.Y. Li, Y.A. Yuan, H.W. Wang, Environ. Sci. Technol. 37 (2003) 292.

[28] C.P. Chu, D.J. Lee, Chem. Eng. Sci. 59 (2004) 1875.

[29] C.P. Chu, D.J. Lee, J. Colloid Interface Sci. 277 (2004) 387.

[30] C.P. Chu, D.J. Lee, J. Chin. Inst. Chem. Eng. 35 (2004) 659.

[31] C.P. Chu, D.J. Lee, X.F. Peng, Water Res. 38 (2004) 2125. 
[32] C.P. Chu, D.J. Lee, J.H. Tay, Chem. Eng. Sci. 60 (2005) 565.

[33] I.L. Chang, C.P. Chu, D.J. Lee, C.P. Huang, J. Colloid Interface Sci. 185 (1997) 335 .

[34] N. Otsu, IEEE Trans. Syst. Man Cybernet. 9 (1979) 62.
[35] T. Tao, X.F. Peng, D.J. Lee, J. Chin. Inst. Chem. Eng. 36 (2005) 511. [36] T. Tao, X.F. Peng, D.J. Lee, J. Chin. Inst. Chem. Eng. 37 (2006) 299. [37] Q. Jiang, B.E. Logan, Environ. Sci. Technol. 25 (1991) 2031. [38] M.R. Wiesner, Water Res. 26 (1992) 379. 